\title{
Lu-, Sm- and Gd-doped ceria: a comparative approach to their structural properties
}

Cristina Artini ${ }^{a, b, ~ *}$, Marcella Pani ${ }^{a, c}$, Maria Maddalena Carnasciali ${ }^{a, d}$, Jasper Rikkert Plaisier ${ }^{e}$, Giorgio Andrea Costa ${ }^{a, c}$

a DCCI, Department of Chemistry and Industrial Chemistry, University of Genova, Via Dodecaneso 31, 16146 Genova, Italy

${ }^{\mathrm{b}}$ CNR-ICMATE, Via De Marini 6, 16149 Genova, Italy

${ }^{\mathrm{c}}$ CNR-SPIN Genova, Corso Perrone 24, 16152 Genova, Italy

d INSTM, Genova Research Unit, Via Dodecaneso 31, 16146 Genova, Italy

${ }^{\mathrm{e}}$ Elettra - Sincrotrone Trieste S.C.p.A., ss 14, km 163.5, 34149 Basovizza, Trieste, Italy 


\section{Rietveld refinement plots}

In this file all the Rietveld plots obtained after the last refinement cycle of samples belonging to the $\left(\mathrm{Ce}_{1-x} \mathrm{Lu}_{x}\right) \mathrm{O}_{2-x / 2}$ system are reported. The dotted and the continuous line are the experimental and the calculated diffractogram, respectively; the lower line is the difference curve; vertical lines indicate the position of the expected peaks.

Samples with $x$ ranging between 0.1 and 0.9 are hereafter named Lu10, Lu20, and so on, according to the atomic percent amount of $\mathrm{RE}^{3+}$ with respect to the total cationic content. 


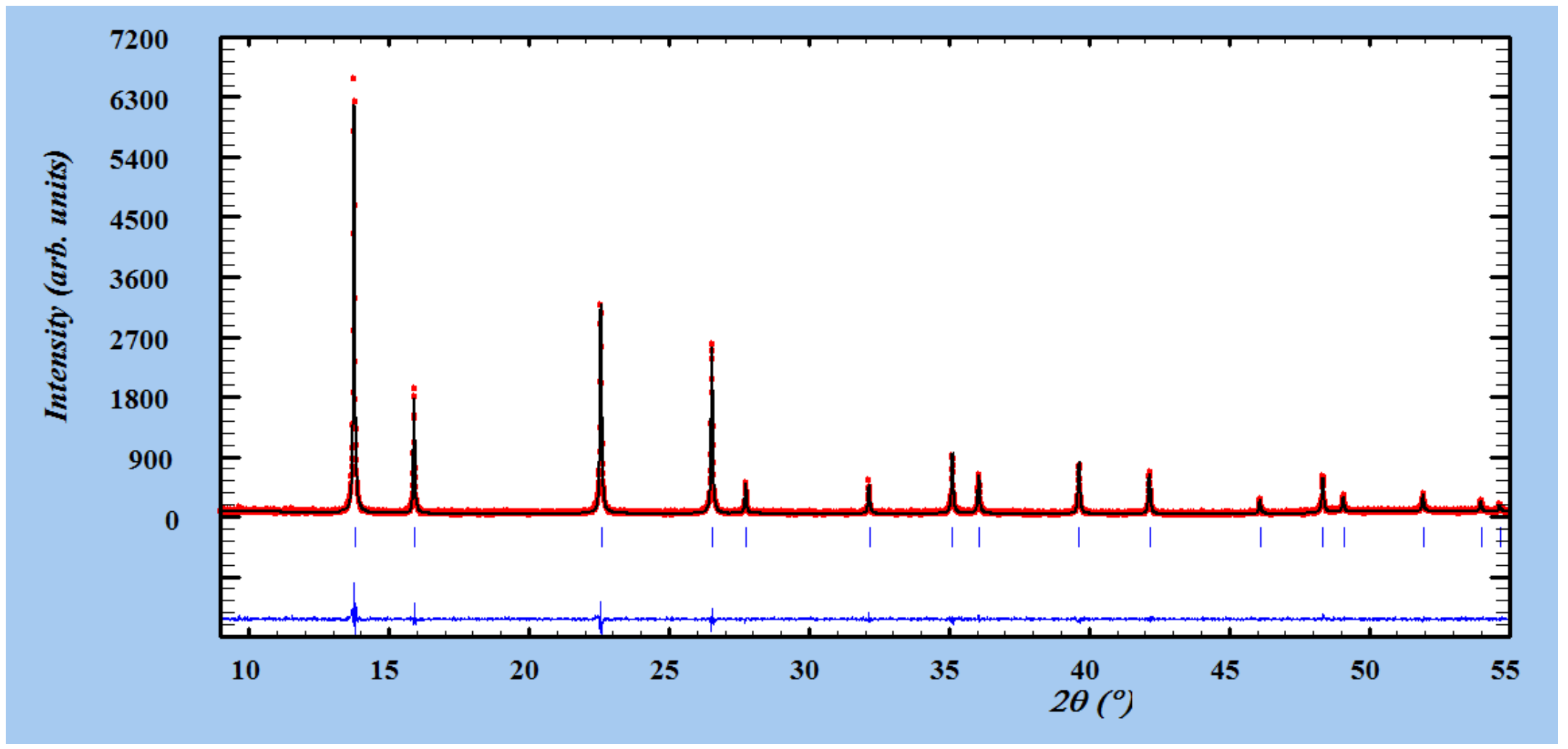

Figure S1 - Rietveld refinement plot for sample $\mathrm{CeO}_{2}$. 


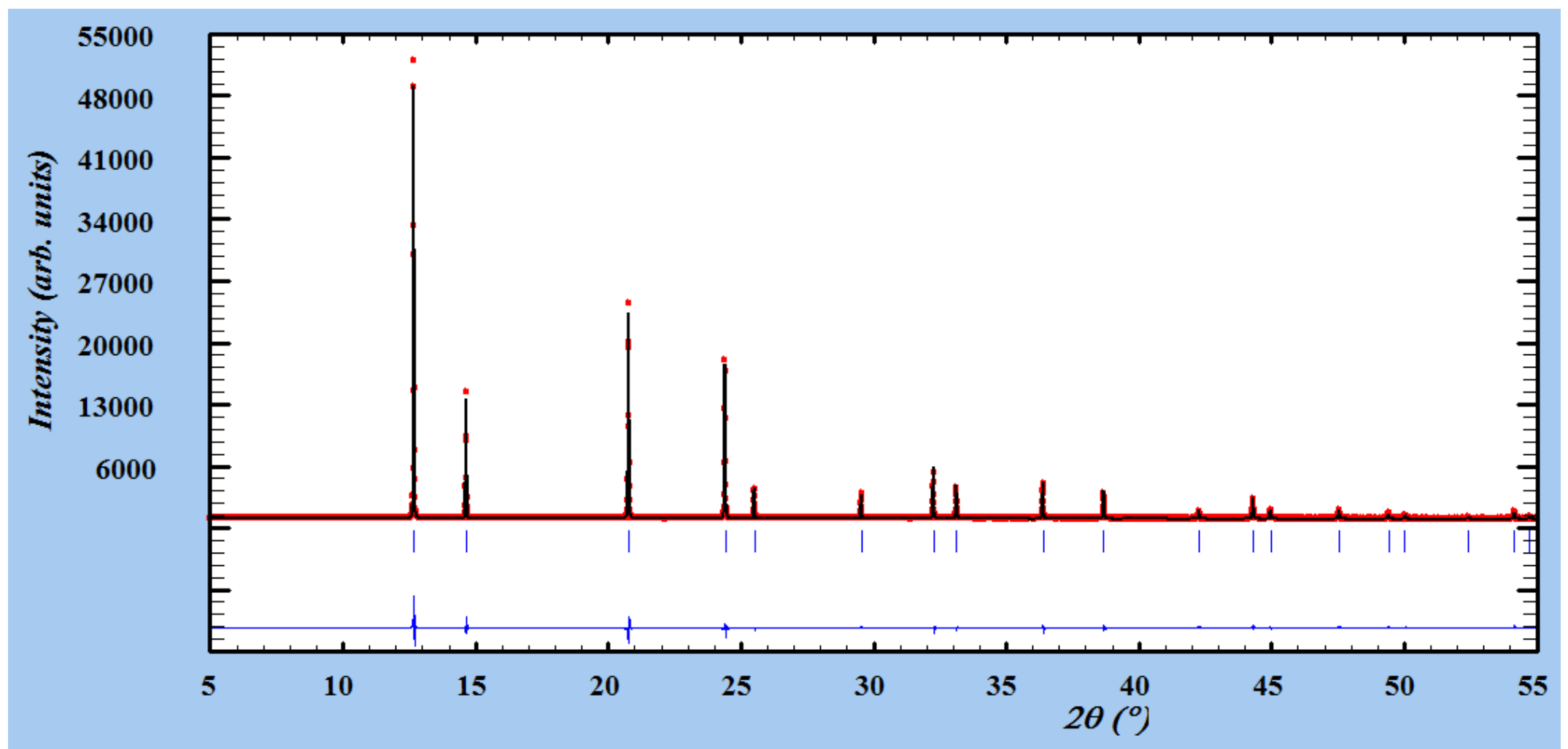

Figure S2 - Rietveld refinement plot for sample Lu10. 


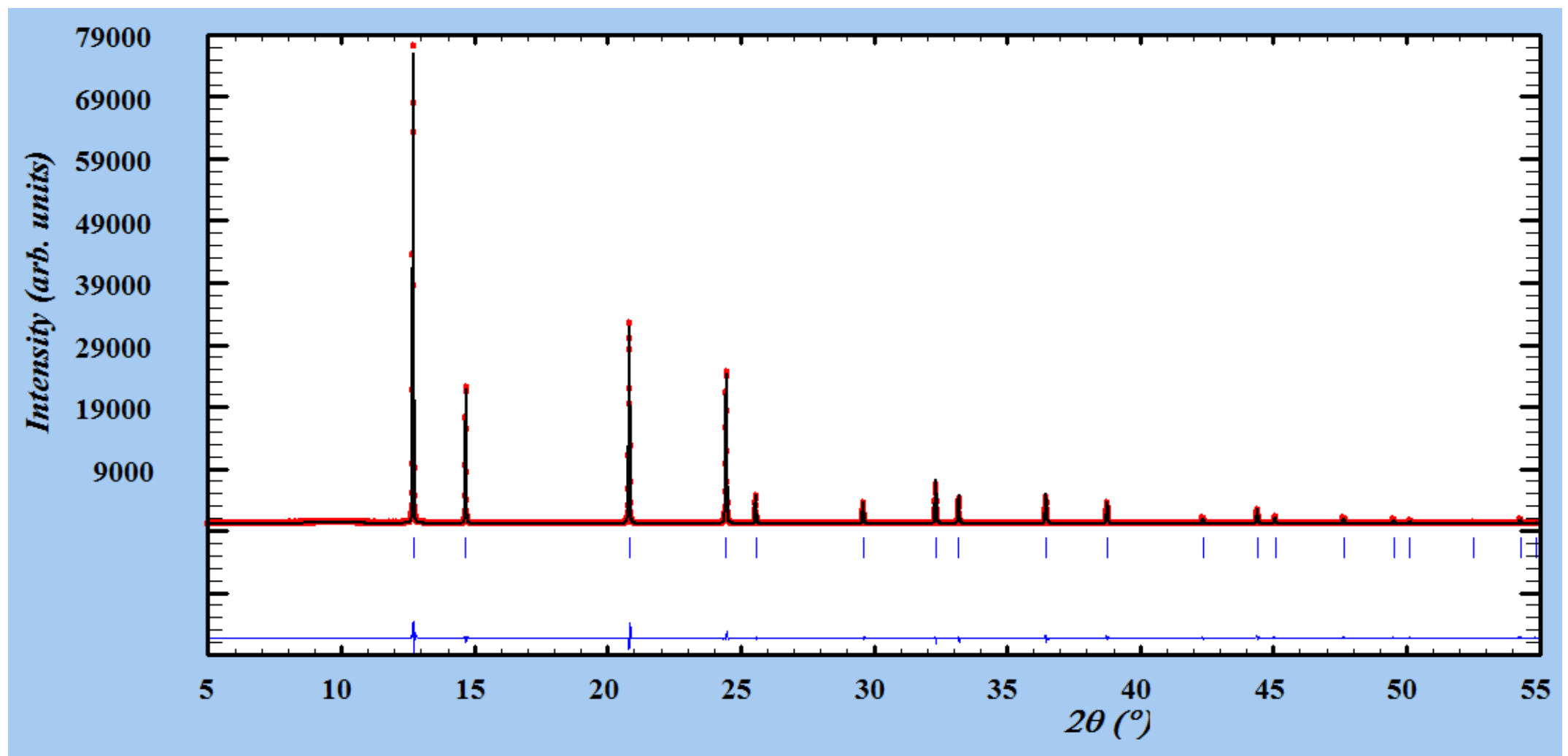

Figure S3 - Rietveld refinement plot for sample Lu20. 


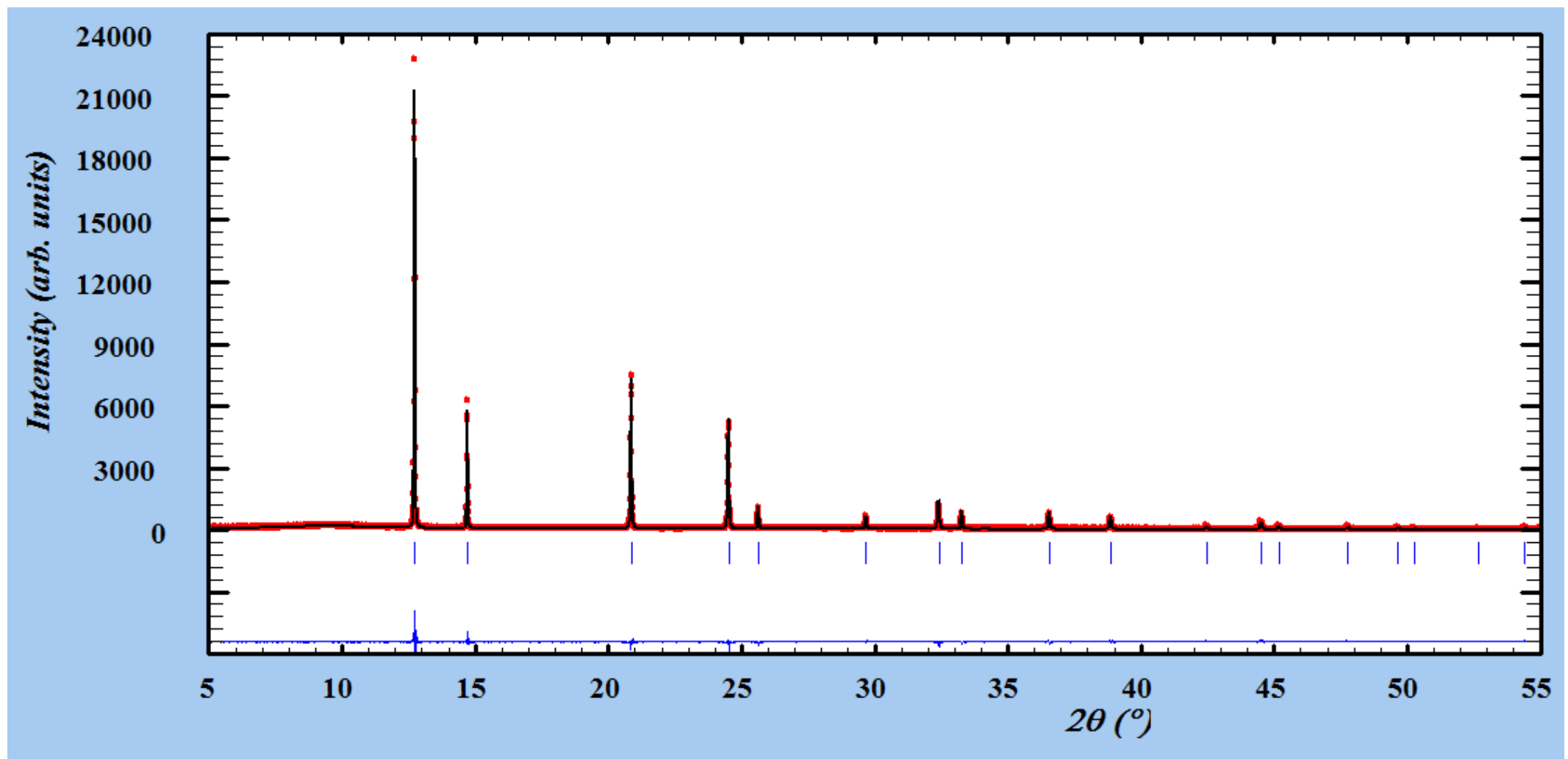

Figure S4 - Rietveld refinement plot for sample Lu30. 


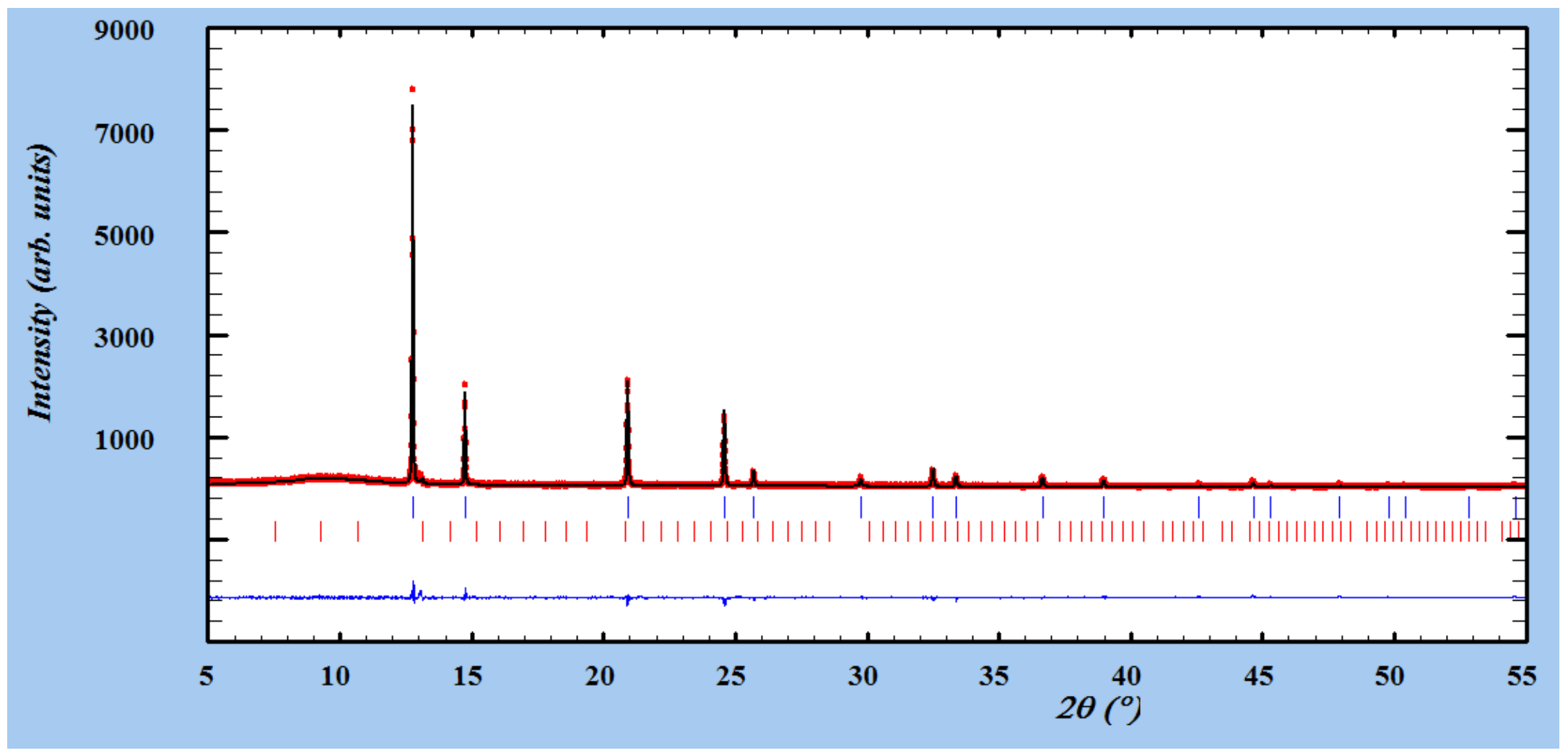

Figure S5 - Rietveld refinement plot for sample Lu40. Blue and red vertical lines indicate the expected peak position of the $\mathrm{F}$ and the $\mathrm{C}$ phase, respectively. 


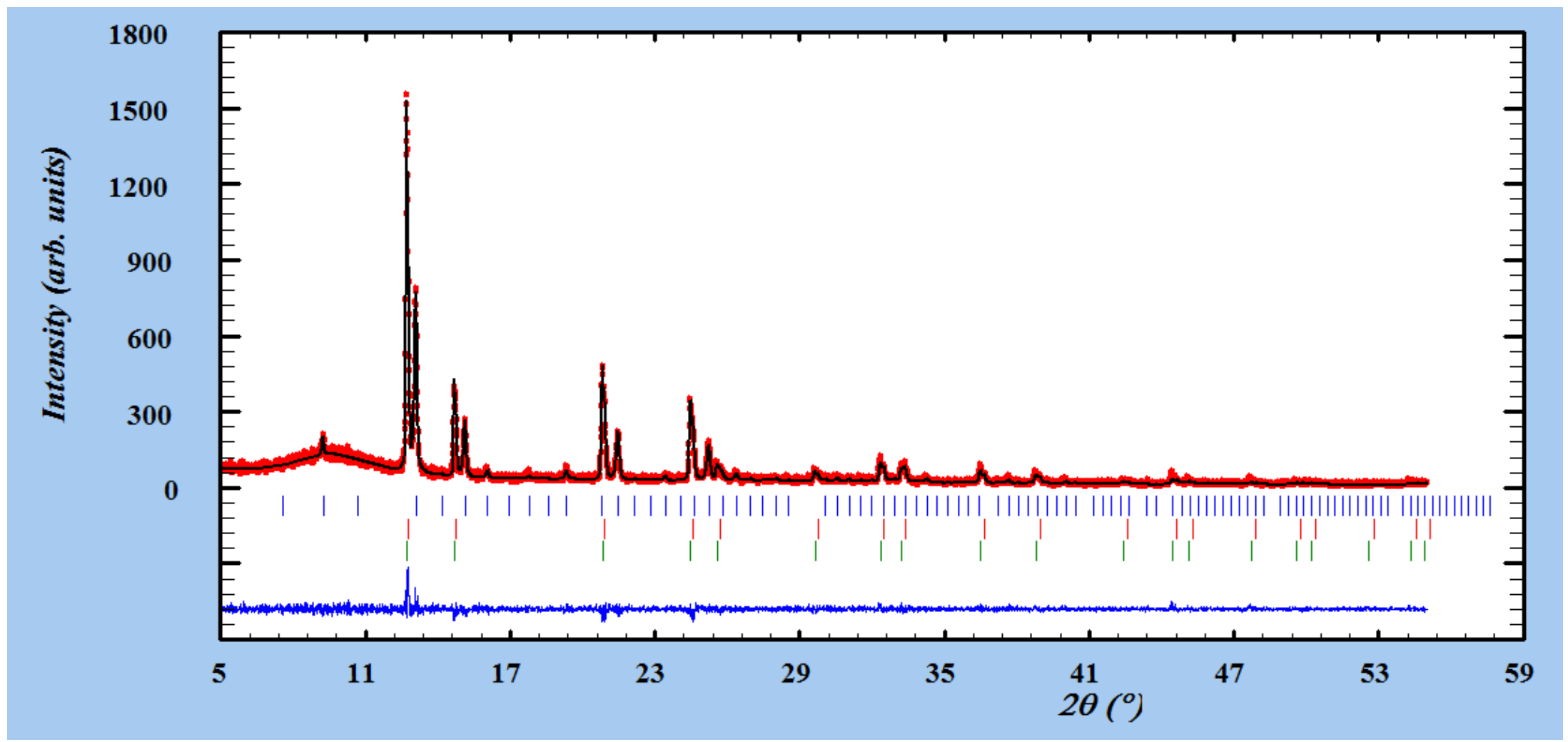

Figure S6 - Rietveld refinement plot for sample Lu50. Blue, red and green vertical lines indicate the expected peak position of the C, F1 and F2 phase, respectively. 


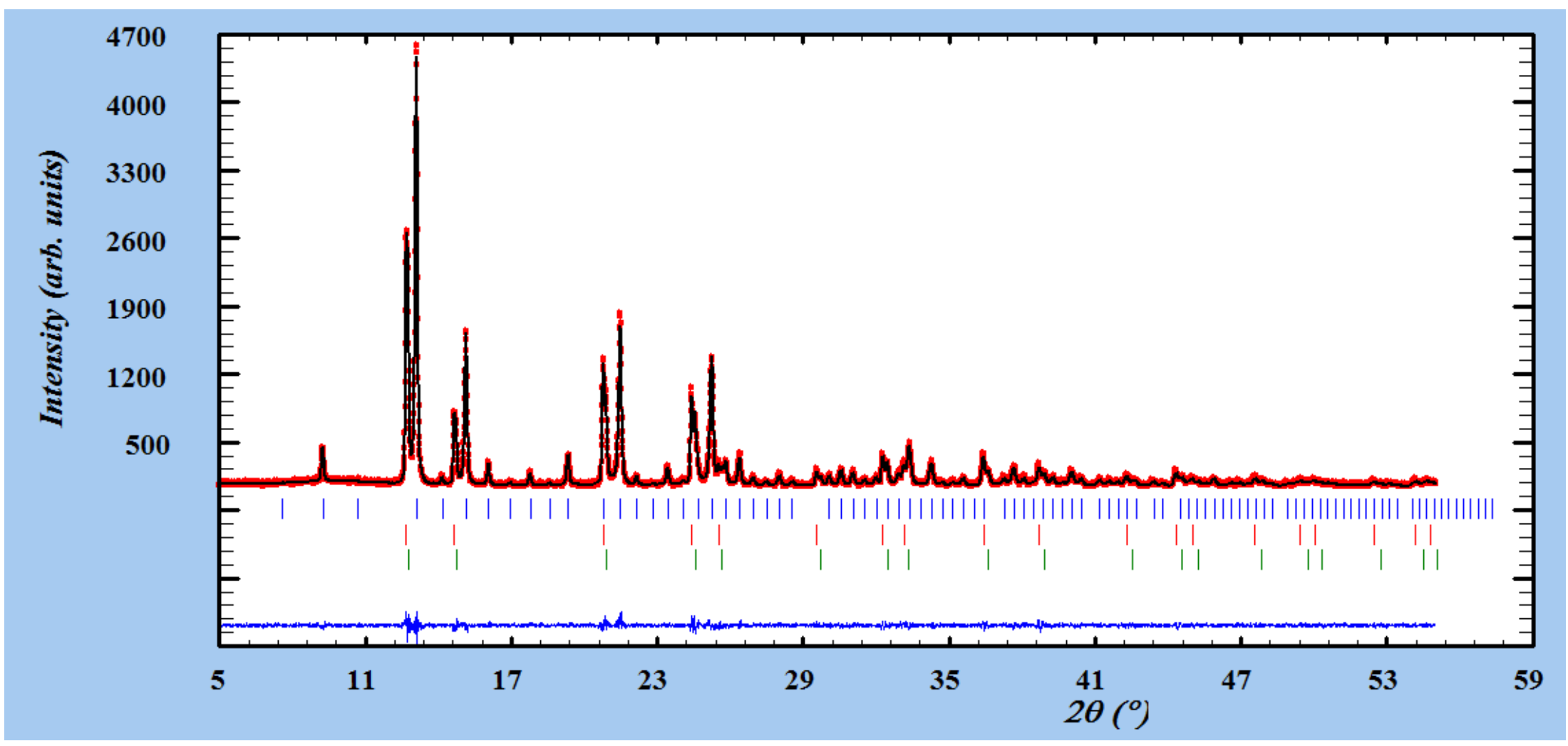

Figure S7 - Rietveld refinement plot for sample Lu60. Blue, red and green vertical lines indicate the expected peak position of the C, F1 and F2 phase, respectively. 


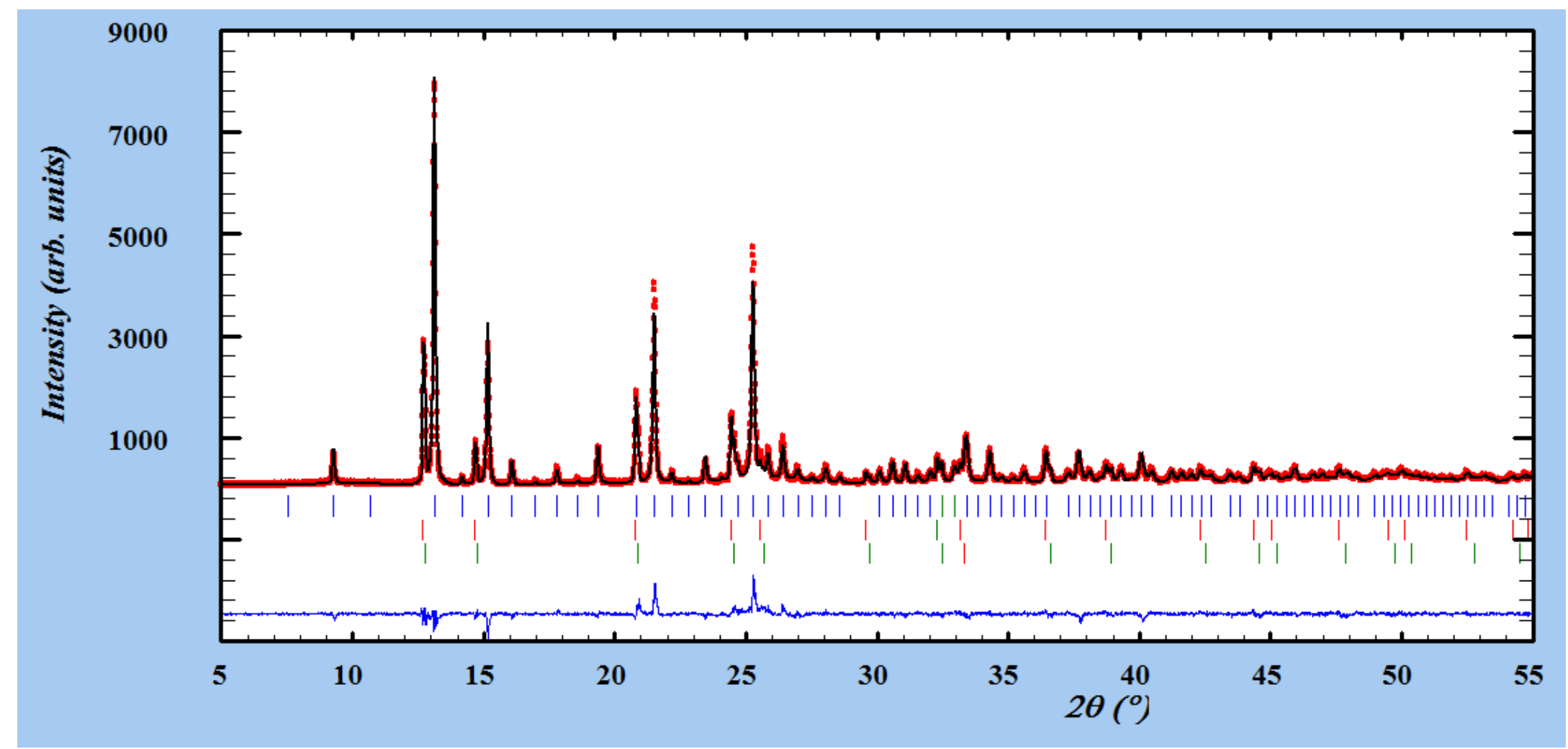

Figure S8 - Rietveld refinement plot for sample Lu70. Blue, red and green vertical lines indicate the expected peak position of the C, F1 and F2 phase, respectively. 


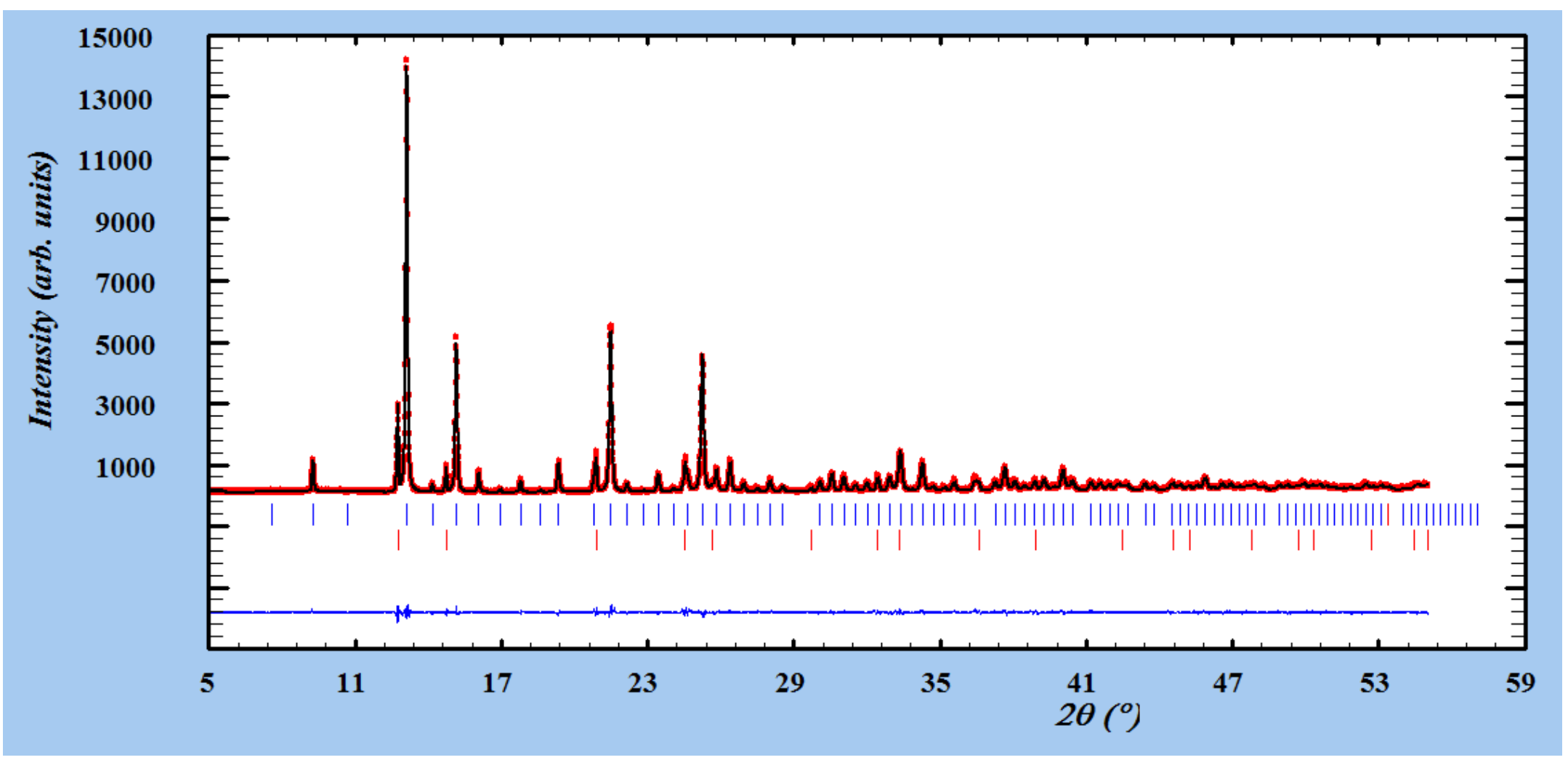

Figure S9 - Rietveld refinement plot for sample Lu80. Blue and red vertical lines indicate the expected peak position of the $\mathrm{C}$ and the $\mathrm{F}$ phase, respectively. 


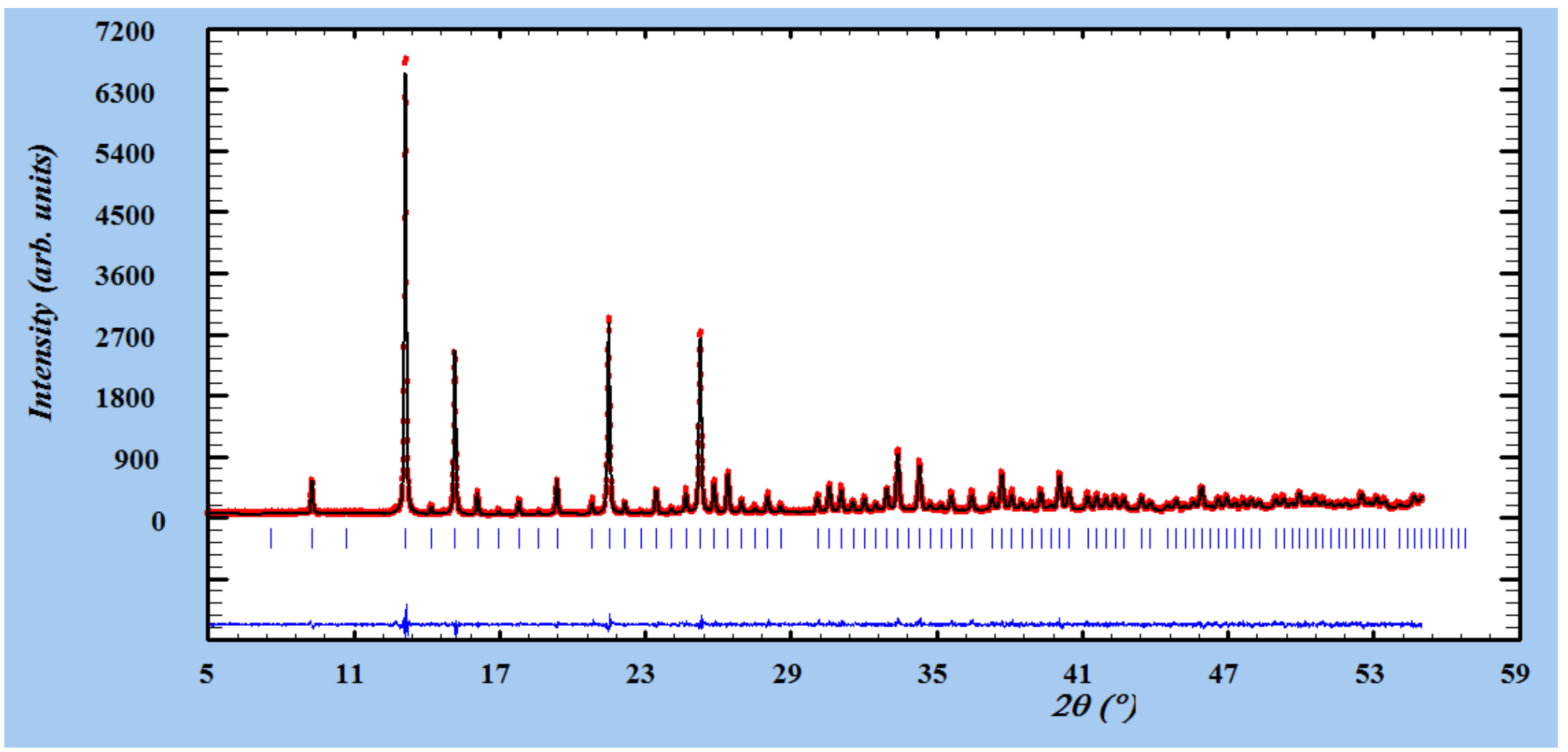

Figure S10 - Rietveld refinement plot for sample Lu90. 


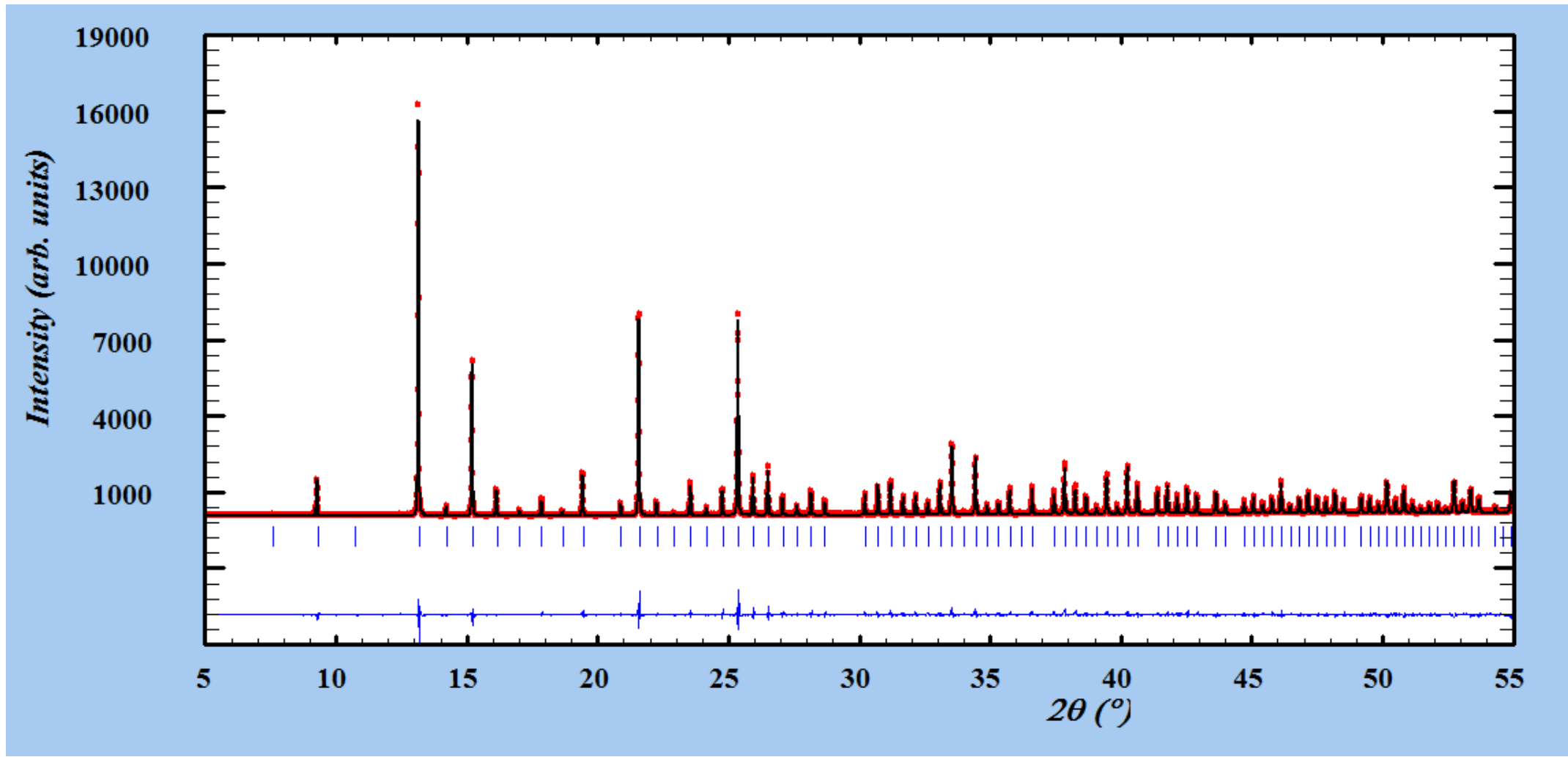

Figure S11 - Rietveld refinement plot for sample $\mathrm{Lu}_{2} \mathrm{O}_{3}$. 
Table $\mathrm{S} 1$ - Refined $\mathrm{B}_{\mathrm{ov}}$ values of both phases, and refined atomic positions of the $\mathrm{C}$ phase.

\begin{tabular}{|c|c|c|c|c|c|c|c|}
\hline \multirow{2}{*}{ Sample } & $\mathbf{F}$ & \multicolumn{6}{|c|}{$\mathbf{C}$} \\
\hline & $\mathbf{B}_{\mathrm{ov}}$ & $\mathbf{B}_{\text {ov }}$ & $\mathbf{X}_{\mathrm{Lu1} / \mathrm{Ce} 1}$ & $\mathbf{x}_{\mathbf{O 1}}$ & yo1 & $\mathbf{z}_{\mathbf{O 1}}$ & $\mathbf{x}_{\mathrm{O} 2}$ \\
\hline $\mathrm{CeO}_{2}$ & $0.95(1)$ & - & - & - & - & - & - \\
\hline Lu10 & $1.60(3)$ & - & - & - & - & - & - \\
\hline Lu20 & $1.94(2)$ & - & - & - & - & - & - \\
\hline Lu30 & $2.85(4)$ & - & - & - & - & - & - \\
\hline Lu40 & $4.15(9)$ & not refined & not refined & not refined & not refined & not refined & not refined \\
\hline$L u 50$ & $2.94(7)$ & $3.8(2)$ & $0.2789(6)$ & $0.110(7)$ & $0.362(9)$ & $0.13(1)$ & $0.11(9)$ \\
\hline$L u 60$ & $2.37(4)$ & $2.34(4)$ & $0.2803(1)$ & $0.100(1)$ & $0.360(1)$ & $0.131(2)$ & $0.13(3)$ \\
\hline Lu70 & $1.19(4)$ & $0.79(3)$ & $0.28017(9)$ & $0.095(1)$ & $0.360(1)$ & $0.132(1)$ & $0.161(5)$ \\
\hline$L u 80$ & $1.52(6)$ & $0.69(2)$ & $0.27990(5)$ & $0.1008(8)$ & $0.3562(8)$ & $0.129(1)$ & $0.155(3)$ \\
\hline Lu90 & - & $0.80(2)$ & $0.28015(6)$ & $0.097(1)$ & $0.355(1)$ & $0.132(1)$ & $0.14(1)$ \\
\hline $\mathrm{Lu}_{2} \mathrm{O}_{3}$ & - & $0.53(2)$ & $0.28297(5)$ & $0.0958(8)$ & $0.3572(9)$ & $0.1291(9)$ & - \\
\hline
\end{tabular}


Table S2 - Peak frequencies of the Raman mode at $\sim 465 \mathrm{~cm}^{-1}$.

\begin{tabular}{|c|c|c|}
\hline Sample & Point & $\begin{array}{c}\text { Peak position } \\
\text { of mode at } \\
\sim 465 \mathrm{~cm}^{-1}\end{array}$ \\
\hline $\mathrm{CeO}_{2}$ & 1 & $461.52(3)$ \\
\hline \multirow[t]{3}{*}{ Lu10 } & 1 & $463.50(5)$ \\
\hline & 2 & $464.94(4)$ \\
\hline & 3 & $462.86(4)$ \\
\hline \multirow[t]{3}{*}{ Lu20 } & 1 & $467.65(5)$ \\
\hline & 2 & $467.44(7)$ \\
\hline & 3 & $466.31(7)$ \\
\hline \multirow[t]{3}{*}{ Lu30 } & 1 & $471.25(8)$ \\
\hline & 2 & $472.30(9)$ \\
\hline & 3 & $472.25(9)$ \\
\hline \multirow[t]{3}{*}{ Lu40 } & 1 & $483.5(2)$ \\
\hline & 2 & $481.4(1)$ \\
\hline & 3 & $478.9(1)$ \\
\hline \multirow[t]{6}{*}{ Lu50 } & 1 & $468.90(7)$ \\
\hline & 2 & $469.13(6)$ \\
\hline & 3 & $469.23(6)$ \\
\hline & 4 & $472.04(9)$ \\
\hline & 5 & $473.8(1)$ \\
\hline & 6 & $477.2(1)$ \\
\hline \multirow[t]{7}{*}{ Lu60 } & 1 & $467.86(4)$ \\
\hline & 2 & $467.94(4)$ \\
\hline & 3 & $468.28(4)$ \\
\hline & 4 & $468.22(3)$ \\
\hline & 5 & $467.7(1)$ \\
\hline & 6 & $467.4(1)$ \\
\hline & 7 & $469.6(1)$ \\
\hline \multirow[t]{4}{*}{ Lu70 } & 1 & $467.06(3)$ \\
\hline & 2 & $467.37(5)$ \\
\hline & 3 & $467.09(4)$ \\
\hline & 4 & 467.07(4) \\
\hline \multirow[t]{7}{*}{ Lu80 } & 1 & $470(1)$ \\
\hline & 2 & $470(1)$ \\
\hline & 3 & $469(1)$ \\
\hline & 4 & $470(1)$ \\
\hline & 5 & 471(1) \\
\hline & 6 & $470(1)$ \\
\hline & 7 & $469(1)$ \\
\hline \multirow[t]{2}{*}{ Lu90 } & 1 & $470(1)$ \\
\hline & 2 & $470(1)$ \\
\hline
\end{tabular}

